\title{
Nonrelativistic variationally optimized exchange potentials for Ne-like atomic ions having large atomic number
}

\author{
I. A. Howard, ${ }^{1}$ N. H. March, ${ }^{1,2}$ and J. D. Talman ${ }^{3}$ \\ ${ }^{1}$ Department of Physics, University of Antwerp, Groenenborgerlaan 171, Antwerp, Belgium \\ ${ }^{2}$ Oxford University, Oxford, England \\ ${ }^{3}$ Department of Applied Mathematics, University of Western Ontario, London, Ontario, Canada \\ (Received 8 April 2003; revised manuscript received 17 July 2003; published 20 October 2003)
}

\begin{abstract}
In an earlier study, the exchange energy density $\epsilon_{x}(r)$ has been calculated analytically for Ne-like atomic ions in the nonrelativistic limit of large atomic number $Z$. Here, though now by numerical methods based on the optimized exchange potential approximation, corresponding results are obtained for $V_{x}(r)$ for such tenelectron ions at large $Z$. The approach to the bare Coulomb limiting form of the $1 / Z$ expansion is considered in some detail, as are corrections to the Slater potential $V_{x}^{S l}(r)$, given in terms of the exchange energy density and ground-state density $\rho(r)$ by $2 \epsilon_{x}(r) / \rho(r)$.
\end{abstract}

DOI: 10.1103/PhysRevA.68.044502

PACS number(s): 31.15.Ew

\section{BACKGROUND AND OUTLINE}

In recent work, Howard et al. [1] have calculated analytically the nonrelativistic limiting form of the exchange energy density for the Ne-like series of positive atomic ions in the limit of large atomic number. These authors defined the exchange energy density $\epsilon_{x}(r)$ from the Dirac [2] result

$$
E_{x}=-\frac{e^{2}}{4} \int \frac{\gamma^{2}\left(\mathbf{r}, \mathbf{r}^{\prime}\right)}{\left|\mathbf{r}-\mathbf{r}^{\prime}\right|} d \mathbf{r} d \mathbf{r}^{\prime}
$$

where $E_{x}$ is the total exchange energy and $\gamma\left(\mathbf{r}, \mathbf{r}^{\prime}\right) / 2$ is the idempotent form of the Dirac density matrix. It then seems natural, though not of course unique [3], to define the exchange energy density $\epsilon_{x}(r)$ as

$$
\boldsymbol{\epsilon}_{x}(r)=-\frac{e^{2}}{4} \int \frac{\gamma^{2}\left(\mathbf{r}, \mathbf{r}^{\prime}\right)}{\left|\mathbf{r}-\mathbf{r}^{\prime}\right|} d \mathbf{r}^{\prime} .
$$

Evidently, from Eqs. (1.1) and (1.2),

$$
E_{x}=\int \epsilon_{x}(r) d \mathbf{r} .
$$

In Ref. [1], the exchange energy density $\epsilon_{x}(r)$ is given analytically for the ten-electron Ne-like series of positive atomic ions in the nonrelativistic limit of large atomic number $Z$. In particular, it was shown in Ref. [1] that $E_{x}$ is the sum of three terms

$$
E_{x}=E_{x}^{(1)}+E_{x}^{(2)}+E_{x}^{(3)},
$$

but only one of these, $E_{x}^{(1)}$, is known explicitly in terms of the electron density $\rho(r)$. This has the form

$$
E_{x}^{(1)}=-\frac{5 \pi}{3} e^{2} \int r^{2} \rho^{2}(r) d \mathbf{r} .
$$

Both $E_{x}^{(2)}$ and $E_{x}^{(3)}$ are, from the theorems of density functional theory [4], functionals of the ground-state density $\rho(r)$ but contain the kinetic-energy density $t(r)$. This presents problems when one attempts to construct the exchange potential $V_{x}(r)$ as the functional derivative

$$
V_{x}(r)=\frac{\delta E_{x}[\rho]}{\delta \rho} .
$$

Therefore, in Ref. [1], though $\epsilon_{x}(r)$ is given explicitly, $V_{x}(r)$ is approximated by (i) the Slater [5] potential $V_{x}^{S l}(r)$ and (ii) the Harbola-Sahni [6] potential $V_{x}^{H S}(r)$. The former potential, following Slater, is given in terms of $\epsilon_{x}(r)$ and $\rho(r)$ by

$$
V_{x}^{S l}(r)=2 \frac{\epsilon_{x}(r)}{\rho(r)},
$$

and hence in this approximation the functional differentiation in Eq. (1.6) is bypassed. Given $\gamma\left(\mathbf{r}, \mathbf{r}^{\prime}\right)$, the Harbola-Sahni [6] potential is known explicitly and again no functional differentiation is involved. Using the limiting large $Z$ form of Ref. [1] for $\gamma$ (bare Coulomb form, without electron-electron interaction "corrections") both the potentials $V_{x}^{S l}(r)$ and $V_{x}^{H S}(r)$ were plotted as functions of $r$ for $Z=92$ in Ref. [1]. One had no check on the errors of these two approximations, nor on whether at $Z=92$ the limiting form of large $Z$ was already quantitative. These two aspects are now addressed in this Brief Report.

The outline of the present study is then as follows. In Sec. II, the investigations of Sharp and Horton [7] and Talman and Shadwick [8] are first discussed. Then, using the socalled optimized exchange potential, the program of Talman [9] is used to obtain a very accurate approximation to $V_{x}(r)$ for the ten-electron atomic ions. The bare Coulomb limit of this variationally optimized method is also extracted, as well as results for $V_{x}(r)$ for such ions with $Z=50$ and $Z=92$. Then in Sec. III, some consequences of these exchange potentials are summarized, as well as the forces $-\partial V_{x} / \partial r$ in these cases and in the limiting large $Z$ form. In this section, the virial-like relation of Levy and Perdew [10] is tested on the optimized exchange potentials. Finally in Sec. IV, the "closed-shell" Ar-like atomic ions are discussed more briefly, and the range of validity of the large $Z$ limit is the 


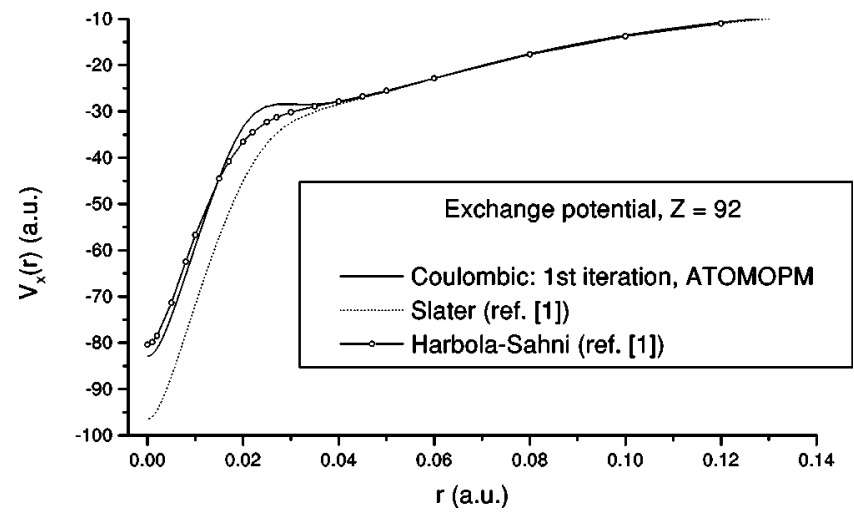

FIG. 1. Limiting form of the optimized exchange potential $V_{x}(r)$ calculated from Eq. (2.1) in the large atomic number $Z$ limit. Curves in this figure are for $Z=92$ and 10 electrons. The result obtained here is compared with the Slater approximation $V_{x}^{S l}(r)$ given in Eq. (1.7) and with the Harbola-Sahni potential $V_{x}^{H S}(r)$. These latter two potentials are taken from Ref. [1].

prime focus here. Section V constitutes a summary, plus some suggestions for future work.

\section{OPTIMIZED EXCHANGE POTENTIALS FOR TEN-ELECTRON ATOMIC IONS FOR LARGE VALUES OF $Z$}

The purpose of this section is to obtain numerical results for the optimized exchange potential for (i) the limit of large $Z$, studied in Ref. [1], and (ii) two positive atomic ions with ten electrons and atomic number $Z$ chosen as 50 and 92. To achieve such results, we made use of the program ATOMOPM of Ref. [9]. The integral equation for the optimized exchange potential $V_{x}(r)$ which we briefly set out below is solved

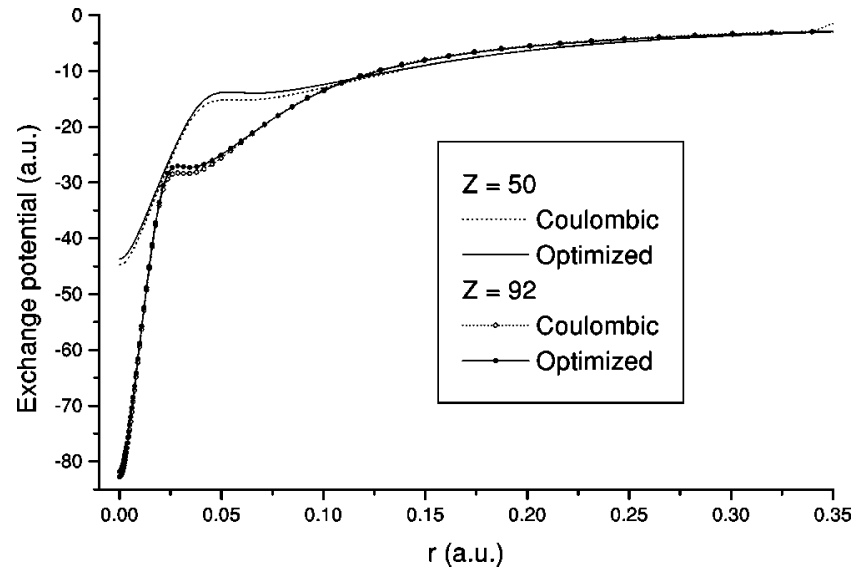

FIG. 2. Illustration of the way $V_{x}(r)$ is obtained by a numerical iterative solution of Eq. (2.1). The fully converged solutions for the optimized exchange potential for nonrelativistic positive atomic ions with ten electrons are shown for both $Z=50$ and $Z=92$. For comparison, the lowest order iteration using the bare Coulomb potential as the starting point is also plotted.

iteratively, starting with bare Coulomb functions. The equation has the form (Eq. (10) of Ref. [9])

$$
\int K\left(\mathbf{r}, \mathbf{r}^{\prime}\right) V_{x}\left(\mathbf{r}^{\prime}\right) d \mathbf{r}^{\prime}=Q(\mathbf{r}) .
$$

Here $K$ involves the one-electron orbitals $\phi_{i}$, and the Green function $G_{i}\left(\mathbf{r}, \mathbf{r}^{\prime}\right)$ for the single-particle Hamiltonian. Each side of Eq. (2.1) is orthogonal to a constant; therefore its solution is arbitrary up to an additive constant, which is determined by the behavior of $V_{x}(\mathbf{r})$ at large $r$. The complete definitions of $K\left(\mathbf{r}, \mathbf{r}^{\prime}\right)$ and $Q(\mathbf{r})$ are given in Ref. [9].

TABLE I. Data from the first step in the iteration of Eq. (2.1) compared with exact Coulomb data for $Z=92$ and 50, ten-electron ions (in a.u.).

\begin{tabular}{lc}
\hline \hline$Z=92$ & $Z=50$ \\
\hline First step, one-electron eigenvalues & \\
$E(1 s)=-4232.004$ & $E(1 s)=-1250.001$ \\
$E(2 s)=-1058.003$ & $E(2 s)=-312.496$ \\
$E(2 p)=-1058.001$ & $E(2 p)=-312.499$ \\
First step, exchange energy & \\
$E_{x}=-160.807$ & $E_{x}=-87.400$ \\
Fully converged values, one-electron eigenvalues & \\
$E(1 s)=-3998.307$ & $E(1 s)=-1125.651$ \\
$E(2 s)=-928.580$ & $E(2 s)=-243.385$ \\
$E(2 p)=-911.984$ & $E(2 p)=-234.858$ \\
Fully converged values, exchange energy & \\
$E_{x}=-155.643$ & $E_{x}=-82.230$ \\
Large-Z limit, one-electron eigenvalues & \\
$E(1 s)=-4232$ & $E(1 s)=-1250$ \\
$E(2 s, 2 p)=-1058$ & $E(2 s, 2 p)=-312.500$ \\
Large-Z limit, exchange energy & \\
$E_{x}=-160.805$ & $E_{x}=-87.394$ \\
\hline \hline
\end{tabular}



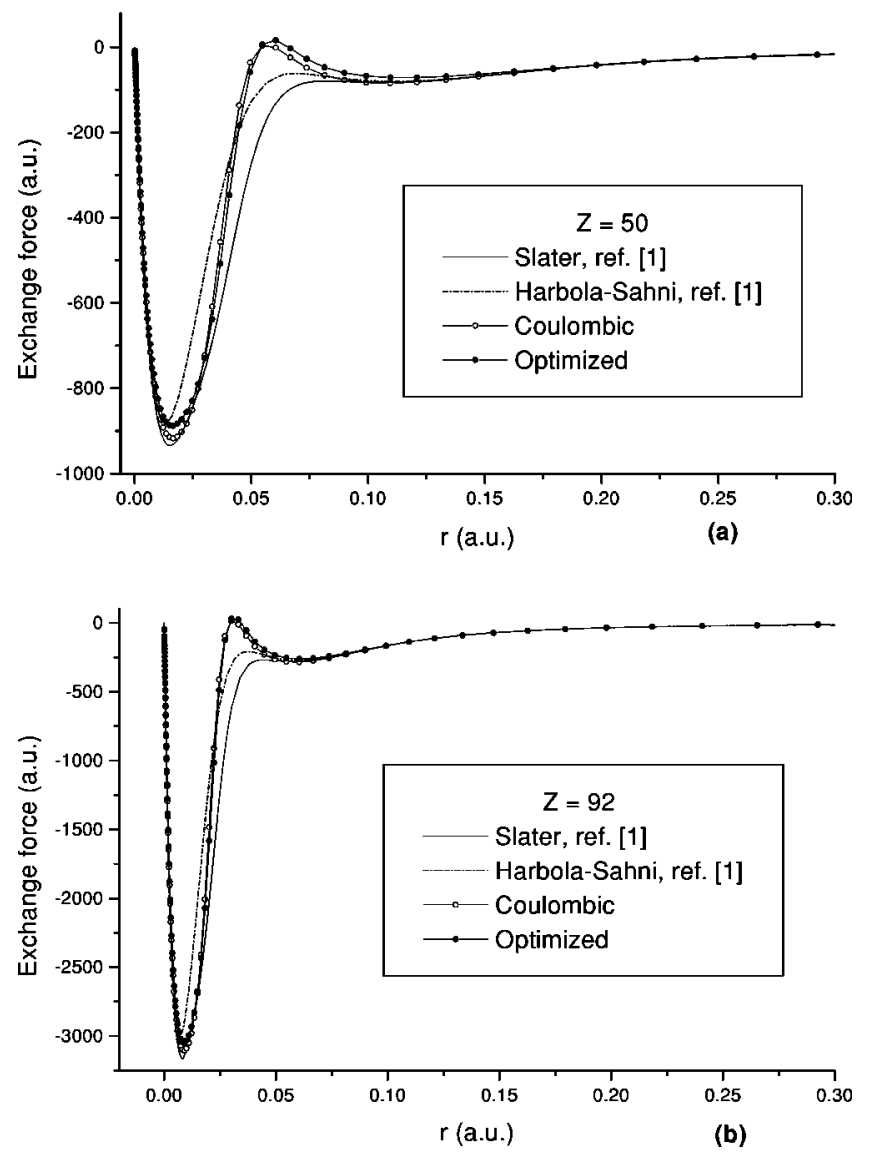

FIG. 3. Limiting large $Z$ (bare Coulomb) form of force $F_{x}(r)$ in Eq. (3.2) for ten electrons. Converged forms of solution of Eq. (2.1) for (a) $Z=50$ and (b) $Z=92$ are shown for comparison. Slater and Harbola-Sahni curves [6] are also plotted.

In the iterative scheme of Ref. [9], it is very convenient that the first step can be taken with the bare Coulomb potential [1]. Of course, to display the result one must choose a specific atomic number. For the choice $Z=92$, we show the result of the program in Ref. [9] for the optimized exchange potential in Fig. 1. For comparison, the results for $V_{x}^{S l}(r)$ and $V_{x}^{H S}(r)$ given in Ref. [1] are reproduced also in Fig. 1. Additionally, we include in Table I some facts pertaining to the first step, to show the accuracy of the numerical program compared with exact Coulomb values.

We expect that $V_{x}(r)$ will be a very accurate numerical approximation to the functional derivative result Eq. (1.6) and thus the appropriate curve in Fig. 1 complements the analytic form of the exchange energy $\epsilon_{x}(r)$ given in Ref. [1] in the limiting bare Coulomb form of the $1 / Z$ expansion. But also using the АТОMOPM program, one can answer for the ten-electron system how large $Z$ must be to validate the bare Coulomb limiting form. This is illustrated in Fig. 2, where the large- $Z$ limiting form shown in Fig. 1 is compared with the fully converged iterative solution of Eq. (2.1) for two values of $Z$, namely, 50 and 92 . It can be seen for the tenelectron case that $Z=92$ is already well approximated by the first step in the iteration of Eq. (2.1), namely, the bare Coulomb limit.

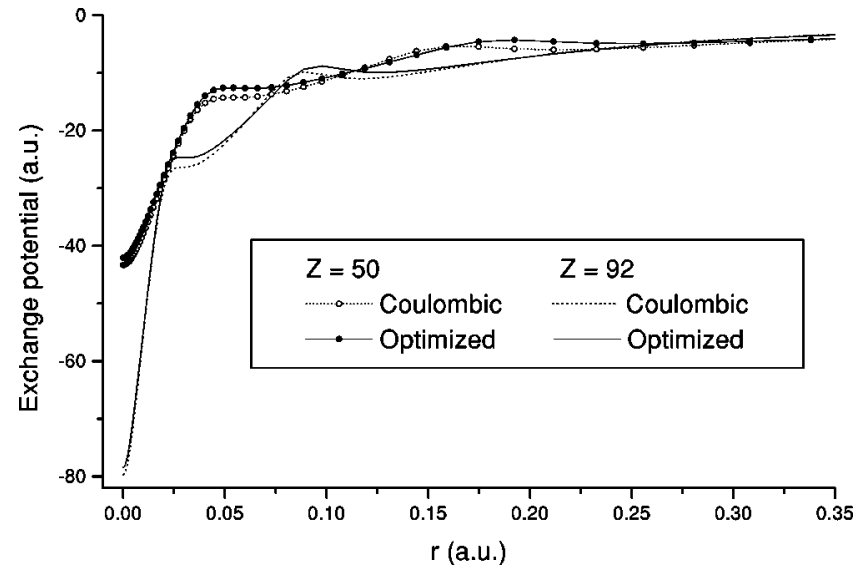

FIG. 4. Fully converged optimized exchange potential $V_{x}(r)$ for the Ar-like series. This is compared with the first iteration using the bare Coulomb potential. Note that the $Z=92$ curves now differ appreciably, in contrast to the close agreement for the ten-electron ions shown in Fig. 2.

\section{SOME OTHER EXCHANGE PROPERTIES: BOTH INTEGRAL AND DIFFERENTIAL}

Turning to other exchange properties, we begin with the virial-like relation of Levy and Perdew [10], which reads

$$
E_{x}=-\int \rho(\mathbf{r}) \mathbf{r} \cdot \nabla V_{x}(\mathbf{r}) d \mathbf{r} .
$$

This relation must be satisfied exactly if $V_{x}$ is the functional derivative of $E_{x}$ given in Eq. (1.6). Therefore a check of the accuracy of Eq. (3.1) is one way to establish the numerical utility of the optimized exchange potential satisfying Eq. (2.1). Since Eq. (3.1) is virial-like, it involves the force $\mathbf{F}_{x}$ associated with the exchange potential $V_{x}$, namely,

$$
\mathbf{F}_{x}=-\nabla V_{x}(\mathbf{r})
$$

The limiting bare Coulomb form of Eq. (3.2) obtained from the first step in the iteration of Eq. (2.1) is compared with the approximations $\mathbf{F}_{x}^{S l}$ and $\mathbf{F}_{x}^{H S}$ obtained in Ref. [1] in the present Fig. 3 for $Z=50$ and 92. We note that these three force approximations all represent zero "exchange force" at the nucleus. All three approximations have the correct large$r$ asymptotic forms

$$
F_{x}=-\frac{e^{2}}{r^{2}}, \quad r \rightarrow \infty .
$$

Our numerical studies for ten electrons show that the LevyPerdew relation Eq. (3.1) is satisfied essentially exactly in both the first iteration (i.e., Coulombic) and in the fully optimized calculation. A related, but somewhat less accurate numerical check was also made in the earlier study of Wang et al. [11].

In concluding this section, we note that the Harbola-Sahni potential $V_{x}^{H S}(r)$ discussed in Ref. [1] (see also Fig. 1) obeys Eq. (3.1) exactly [6] even though, as demonstrated by Levy 


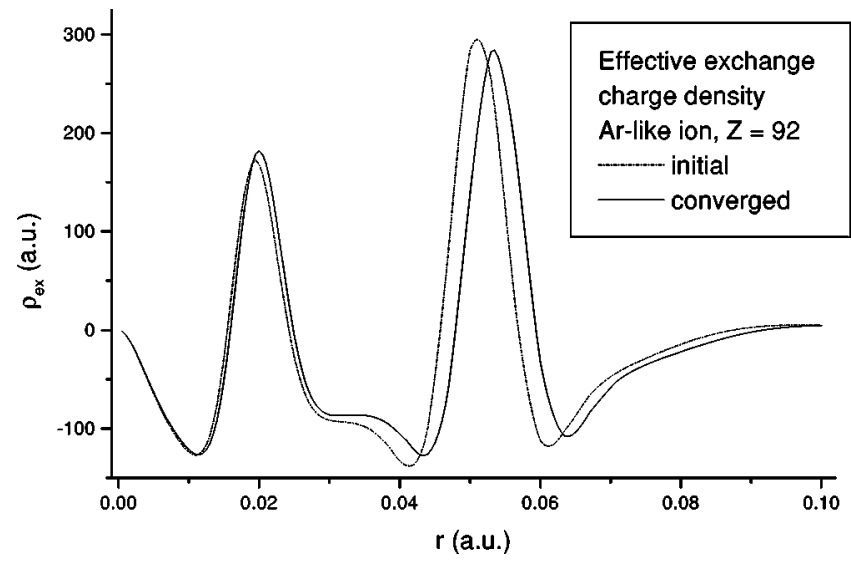

FIG. 5. Radial distribution $r^{2} \rho_{x}(r)$ of an effective exchange charge density $\rho_{x}(r)=-\nabla^{2} V_{x}(r)$ for the Ar-like ion with $Z=92$ and 18 electrons. Results for the initial iteration and for the fully converged optimized exchange potential are compared.

and March [12], "corrections" which are "kinetic" in character are required to convert $V_{x}^{H S}(r)$ to the exact exchangeonly potential.

\section{Ar-LIKE SERIES: BRIEF SUMMARY}

Though, in principle, the work of Ref. [1] in the bare Coulomb limit of large $Z$ could be extended analytically to the 18-electron, Ar-like, series of positive atomic ions, the exchange energy density $\epsilon_{x}(r)$ would be the result, and not $V_{x}(r)$ as explained above for the Ne-like series. Therefore, here we confine ourselves to showing in Fig. 4 the limiting large- $Z$ form, again from the first step in the iteration of Eq. (2.1), for $Z=50$ and 92 . The finally converged curve for the same $Z$ is shown for comparison, again for 18 electrons, in Fig. 4.

Finally, again in response to a suggestion of the referee, we show in Fig. 5 a somewhat different representation of the exchange potential for $Z=92$ and 18 electrons, namely, its Laplacian, related to a charge density $\rho_{e x}(r)$.

\section{SUMMARY AND FUTURE DIRECTIONS}

The main results of the present study are as follows.

(i) The limiting (Coulomb) forms of the first iteration of the optimized effective potential approximation to the exchange potential in the nonrelativistic theory for large atomic number $Z$ for (a) the ten-electron Ne-like positive atomic ions and (b) the 18-electron Ar-like series.

(ii) The converged iterative solutions of Eq. (2.1) for the ten-electron atomic ions for $Z=50$ and $Z=92$. It would, we believe, in the future, be of interest to attempt to use existing theory [13] on the $1 / Z$ expansion for homonuclear diatomic molecules to calculate the limiting forms for some positive molecular ions of the optimized exchange potential $V_{x}(\mathbf{r})$. Possibly the extension to almost spherical $C$ and $B$ cages $[14,15]$ might also prove practicable.

Finally, the referee has drawn our attention to the work of Nagy [16]. She has kindly informed us that if one knows the exact density (say from diffraction or quantum simulation), one can calculate the exchange-correlation potential either with the Zhao-Morrison-Parr [17] method, or other methods, such as her own. In this sense, as the referee pointed out to us, the Zhao-Morrison-Parr approach can be said to be more accurate than the optimized effective potential method. However, the optimized effective potential can be regarded as the exact exchange potential of density-functional theory in the exchange-only approximation $[16,18]$.

\section{ACKNOWLEDGMENTS}

Research by J.D.T. was supported by the Natural Sciences and Engineering Research Council of Canada. I.A.H. acknowledges support from the IWT-Flemish region. N.H.M. wishes to acknowledge partial financial support from the Office of Naval Research for work on density-functional theory. Special thanks are due to Dr. P. Schmidt of that office for much motivation and encouragement.
[1] I.A. Howard, N.H. March, P. Senet, and V.E. Van Doren, Phys. Rev. A 62, 062512 (2000).

[2] P.A.M. Dirac, Proc. Cambridge Philos. Soc. 26, 376 (1930).

[3] See, for example, K. Burke, F.G. Cruz, and K.-C. Lam, J. Chem. Phys. 109, 8161 (1998).

[4] P.C. Hohenberg and W. Kohn, Phys. Rev. 136, B864 (1964).

[5] J.C. Slater, Phys. Rev. 81, 385 (1951).

[6] M.K. Harbola and V. Sahni, Phys. Rev. Lett. 62, 489 (1989).

[7] R.T. Sharp and G.K. Horton, Phys. Rev. 90, 316 (1955).

[8] J.D. Talman and W.F. Shadwick, Phys. Rev. A 14, 36 (1976).

[9] J.D. Talman, Comput. Phys. Commun. 54, 85 (1989).

[10] M. Levy and J.P. Perdew, Phys. Rev. A 32, 2010 (1985).

[11] Y. Wang, J.P. Perdew, J.A. Chevary, L.D. MacDonald, and S.H. Vosko, Phys. Rev. A 41, 78 (1990).
[12] M. Levy and N.H. March, Phys. Rev. A 55, 1885 (1997).

[13] N.H. March and R.G. Parr, Proc. Natl. Acad. Sci. U.S.A. 77, 6285 (1980).

[14] C. Amovilli, I.A. Howard, D.J. Klein, and N.H. March, Phys. Rev. A 66, 013210 (2002).

[15] C. Amovilli and N.H. March, Chem. Phys. Lett. 347, 459 (2001).

[16] A. Nagy, Phys. Rep. 298, 1 (1998); A. Nagy, J. Phys. B 26, 43 (1993); A. Nagy, Philos. Mag. B 69, 779 (1994).

[17] Q. Zhao, R.C. Morrison, and R.G. Parr, Phys. Rev. A 50, 2138 (1994).

[18] V. Sahne, J. Gruenebaum, and J.P. Perdew, Phys. Rev. B 26, 4371 (1982). 